\title{
Switched Cooperative Driving Model towards Human Vehicle Copiloting Situation: A Cyberphysical Perspective
}

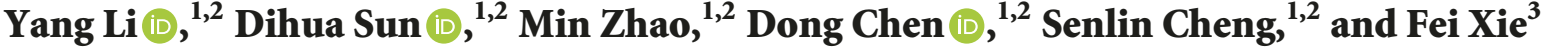 \\ ${ }^{1}$ Key Laboratory of Dependable Service Computing in Cyber Physical Society of Ministry of Education, Chongqing University, \\ Chongqing 400044, China \\ ${ }^{2}$ School of Automation, Chongqing University, Chongqing 400044, China \\ ${ }^{3}$ China Automotive Engineering Research Institute Co., Ltd., Chongqing 401122, China
}

Correspondence should be addressed to Dihua Sun; d3sun@163.com

Received 5 October 2017; Revised 31 December 2017; Accepted 15 January 2018; Published 27 February 2018

Academic Editor: Jose E. Naranjo

Copyright (C) 2018 Yang Li et al. This is an open access article distributed under the Creative Commons Attribution License, which permits unrestricted use, distribution, and reproduction in any medium, provided the original work is properly cited.

\begin{abstract}
Development of highly automated and intelligent vehicles can lead to the reduction of driver workload. However, it also causes the out-of-the-loop problem to drivers, which leaves drivers handicapped in their ability to take over manual operations in emergency situations. This contribution puts forth a new switched driving strategy to avoid some of the negative consequences associated with out-of-the-loop performance by having drivers assume manual control at periodic intervals. To minimize the impact of the transitions between automated and manual driving on traffic operations, a switched cooperative driving model towards human vehicle copiloting situation is proposed by considering the vehicle dynamics and the realistic intervehicle communication in a cyberphysical view. The design method of the switching signal for the switched cooperative driving model is given based on the Lyapunov stability theory with the comprehensive consideration of platoon stability and human factors. The good agreement between simulation results and theoretical analysis illustrates the effectiveness of the proposed methods.
\end{abstract}

\section{Introduction}

Advanced driver assistance systems (ADASs) assist drivers or take over driving tasks to operate vehicles in a safe, efficient, and comfortable way [1]. Many advanced driver assistance systems are cooperative systems, where vehicles are connected with each other via vehicle-to-vehicle $(\mathrm{V} 2 \mathrm{~V})$ communications [2]. The cooperative adaptive cruise control (CACC) system is a promising example of ADAS which enables vehicles to follow one another with short intervehicle distances using intervehicle wireless communication as well as onboard sensors, thus improving road throughput, without compromising safety [3].

The Society of Automotive Engineers (SAE), the National Highway Traffic Safety Administration (NHTSA), and the German Federal Highway Research Institute (BASt) have provided their definitions of "levels of automated driving" [4-6]. Although these definitions are different, the essential criteria that define the different levels of automation are similar in each of the three cases.
The progress of automated driving technology and the development of the ADAS make the problem of human vehicle copiloting a hot topic, which has received a degree of attention in recent years, partly due to the considerable outreach activities undertaken by companies such as Google and Tesla [7].

It is helpful to think of the driving process as a control loop that consists of goal, implementation, and feedback on whether or not the chosen strategy satisfies the desired state. When the driver is in the loop, he/she is actively engaged in all three processes. However, as soon as automation is engaged, the human operator is potentially relegated from one or more of these stages, meaning that they could become out of the loop [8].

Several general conclusions can be derived from previous researches on human-machine cooperation: a greater degree of automation reduces the variability of human performance, but it also leads to "human out-of-the-loop" problems, such as complacency, loss of situation awareness, loss of manual control skills, and behavioral adaptation [9-12]. A literature 
review by De Winter et al. [13] shows that drivers' workload and situation awareness are vastly different for driving with ACC compared to Highly Automated Driving (HAD).

Such human factors issues resulting from increased automation levels need to be taken into consideration when designing automated driving and ADASs $[14,15]$. A driving state model describing the human monitoring level and the allocation of lateral and longitudinal control tasks was introduced. In addition, an authority transition in automated driving is defined as the process of changing from one static state of driving to another static state [16].

Some studies found that the type of functional limitation [17] and drivers' experience of using the automated driving system $[18,19]$ have an effect on the outcome of the takeover, whereas drivers' trust in the automation has been found to not influence the driver's behavior during the takeover $[19,20]$. Further studies have shown that drivers' ability to take over when a transition to manual control is required can be improved by providing information about the limits and reliability of the automation $[21,22]$.

Previous works that detect driver fatigue or distraction in real time have used both eye tracking data and vehicle-based measures such as speed and lateral position [23-25]. A variety of measures have been used to determine the thresholds of such distraction, ranging from average duration of glances away from the road in a $4.3 \mathrm{~s}$ wide sliding window [26] to the percentage of on-road gaze points in a $60 \mathrm{~s}$ sliding window [27].

As the above states, the existing studies about human vehicle copiloting and transition between automated and manual driving mainly focus on the specific switching process and conditions. Many studies are subject to expensive devices to detect drivers' states and vehicle running data, and few researches pay close attention to the impact of the transitions on traffic operations.

In level 3 driving automation defined by SAE, the automated driving system performs all aspects of dynamic driving task. The human driver does not need to monitor the driving environment, which will make drivers out of the loop, but should respond appropriately to take over the vehicle in an emergency situation. Many companies and researchers have recognized the potential problem; the likes of Ford and Waymo believe the handoff is fraught with so many problems; they have elected to eschew such development and pursue fully driverless cars.

Meanwhile, many people neglect the issues in level 2. The task of the human driver turns from operating the vehicle to supervising the status of the automated driving system and traffic environment in level 2. However, the studies on human factors show that human beings are not good at supervising tasks. Boredom, distraction, and fatigue will result in the long time supervising task.

Thus, to avoid some of the negative consequences associated with the factors mentioned above, this paper puts forth a periodic switched driving strategy to keep drivers always in the loop and prevent drivers' distraction and fatigue resulting from the long time supervising task. In order to minimize the impact of the periodic transitions between automated and manual driving on traffic operations,

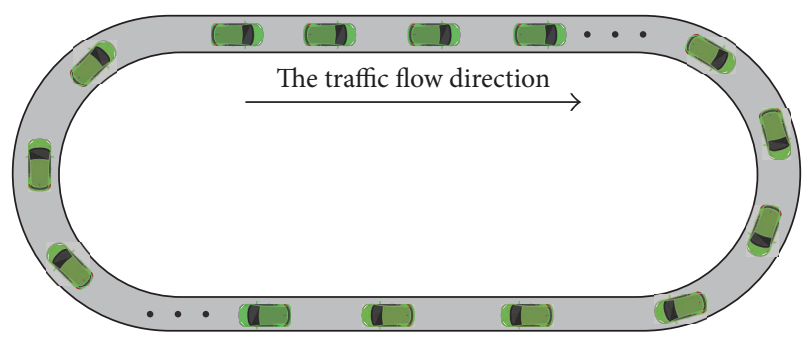

FIgURE 1: The schematic diagram of the vehicle platoon.

a switched cooperative driving model towards human vehicle copiloting situation is proposed by considering the vehicle dynamics and the realistic intervehicle communication in a cyberphysical view. Meanwhile, the design method of the switching signal for the switched cooperative driving model is given based on the Lyapunov stability theory with the comprehensive consideration of platoon stability and human factors.

The remainder of this paper is organized as follows. Section 2 introduces the construction of switched cooperative driving model; stability analysis is given in Section 3; Section 4 presents the design method and suggestion of the switching signal; and numerical examples are provided to illustrate the effectiveness of the proposed methods in Section 5. Section 6 summarizes the main conclusions.

\section{The Switched Cooperative Driving Model}

Consider a platoon of $N$ vehicles which follow one another along a single-lane ring road; as depicted in Figure 1, the vehicle dynamics can be described by the transfer function $G(s)$ in the Laplace domain, with $s \in \mathbb{C}$, according to

$$
G_{n}(s)=\frac{x_{n}(s)}{u_{n}(s)}=\frac{1}{s^{2}\left(T_{n} s+1\right)} e^{-\tau_{n} s},
$$

where $T_{n}$ and $\tau_{n}$ are the mechanical inertia coefficient and time delay of the $n$th follower vehicle in the platoon, respectively. $u_{n}$ is the vehicle input (desired acceleration), whereas the position $x_{n}$ represents the output. Note that $\cdot(s)$ denotes the Laplace transform of the corresponding time-domain variable $\cdot(t)$. Assume that all vehicles are homogeneous; $G(s)$ is identical for all vehicles; this means $T_{1}=T_{2}=\cdots=T_{N}$, $\tau_{1}=\tau_{2}=\cdots=\tau_{N}$.

In this paper, we focus on the modeling of the switched cooperative driving system towards human vehicle copiloting situation; the distinguishing feature between automated and manual driving is the CACC system as an extension of the adaptive cruise control with wireless intervehicle communication, which enables vehicles to obtain the control information of the preceding vehicle. Moreover, the vehicle dynamics and the realistic intervehicle communication should be considered in a cyberphysical perspective. Let $a_{n}(t), v_{n}(t)$, and $x_{n}(t)$ denote the acceleration, velocity, and positon of the $n$th follower vehicle at time $t$. Thus, the vehicleto-vehicle cooperative driving model's dynamics equation 
for automated and manual driving can be formulated as follows:

$$
\begin{aligned}
a_{n}(t)= & -T_{n} \dot{a}_{n}(t) \\
& +k_{n}^{\mathrm{CACC}}\left[V\left(y_{n}\left(t-\tau_{n}\right)\right)-v_{n}\left(t-\tau_{n}\right)\right] \\
& +\lambda_{n}^{\mathrm{CACC}} \Delta v_{n}\left(t-\tau_{n}\right) \\
& +\gamma_{n}^{\mathrm{CACC}} a_{n+1}\left(t-\tau_{n}^{c}-\tau_{n}\right) \\
& \quad \text { for automated driving } \\
a_{n}(t)= & -T_{n} \dot{a}_{n}(t)+k_{n}^{\text {hum }}\left[V\left(y_{n}\left(t-\tau_{n}\right)\right)-v_{n}\left(t-\tau_{n}\right)\right] \\
& +\lambda_{n}^{\text {hum }} \Delta v_{n}\left(t-\tau_{n}\right) \quad \text { for manual driving, }
\end{aligned}
$$

where $k_{n}^{\mathrm{CACC}}, \lambda_{n}^{\mathrm{CACC}}$, and $\gamma_{n}^{\mathrm{CACC}}$ are the sensitivities for automated driving and $k_{n}^{\text {hum }}$ and $\lambda_{n}^{\text {hum }}$ are the sensitivities when vehicles are in manual driving condition. $y_{n}(t)$ and $\Delta v_{n}(t)$ denote the headway and the velocity difference between the $n$th and the $n+1$ th vehicle at time $t$. $\tau_{n}^{c}$ represents the delay of the realistic communication. In this paper, we adopt the optimal velocity function which is carried out by Helbing and Tilch [28]. The optimal velocity function $V(\cdot)$ is based on the hyperbolic tangent function, so it is a monotonically increasing function and has an upper bound, which is defined as

$$
V(x)=V_{1}+V_{2} \tanh \left[C_{1}\left(x-l_{c}\right)-C_{2}\right],
$$

where $V_{1}=6.75 \mathrm{~m} / \mathrm{s}, V_{2}=7.91 \mathrm{~m} / \mathrm{s}, C_{1}=0.13 \mathrm{~m}^{-1}, C_{2}=1.57$, and $l_{c}$ denotes the length of the vehicle.

According to the method of stability analysis from [29, 30], some assumptions should be predefined before detailed discussion.

Assumption 1. All vehicles move at an initial constant velocity when $t \leq 0$.

Assumption 2. All vehicles follow one another without overtaking.

Assumption 3. The 1 th vehicle is the preceding vehicle of the $N$ th vehicle.

Based on the above assumptions, subject to the optimal velocity function, we can easily obtain the following steady state of model (2):

$$
\left[a_{n}^{*}(t), v_{n}^{*}(t), y_{n}^{*}(t)\right]^{T}=\left[0, V\left(\frac{L}{N}\right), \frac{L}{N}\right]^{T},
$$

where

\section{$L$ is the length of the ring road;}

$N$ is the total number of the vehicles.

This implies that, in the steady state, all vehicles run orderly with velocity $V(L / N)$ and headway distance $L / N$.

The objective of this paper is to design an adequate switching signal, not only to keep drivers in the loop, but also to guarantee that the cooperative driving system runs orderly with velocity $V(L / N)$ and headway distance $L / N$ by rejecting the effect of the periodic switch, so as to keep drivers in the loop and effectively suppress the traffic congestion.

Aiming at manual driving scenario, we can obtain the error dynamic of the cooperative driving model by linearization of (2) at the steady state (4) as described in

$$
\begin{aligned}
\delta \dot{a}_{n}(t)= & \frac{k_{n}^{\text {hum }}}{T_{n}}\left[\Lambda \delta y_{n}\left(t-\tau_{n}\right)-\delta v_{n}\left(t-\tau_{n}\right)\right] \\
& +\frac{\lambda_{n}^{\text {hum }}}{T_{n}}\left(\delta v_{n+1}\left(t-\tau_{n}\right)-\delta v_{n}\left(t-\tau_{n}\right)\right) \\
& -\frac{1}{T_{n}} \delta a_{n}(t) \\
\delta \dot{v}_{n}(t)= & \delta a_{n}(t) \\
\delta \dot{y}_{n}(t)= & \delta v_{n+1}(t)-\delta v_{n}(t)
\end{aligned}
$$

where $\delta a_{n}(t)=a_{n}(t)-a_{n}^{*}(t), \delta v_{n}(t)=v_{n}(t)-v_{n}^{*}(t), \delta y_{n}(t)=$ $y_{n}(t)-y_{n}^{*}(t)$, and $\Lambda$ is the slope of the optimal velocity function at $y_{n}(t)=L / N, \Lambda=d V\left(y_{n}(t)\right) /\left.d y_{n}(t)\right|_{y_{n}(t)=L / N}$.

Let $s_{n}(t)=\left[\delta a_{n}(t), \delta v_{n}(t), \delta y_{n}(t)\right]^{T}$; the error dynamic (5) is rewritten as

$$
\begin{aligned}
\dot{s}_{n}(t)= & a_{1} s_{n}(t)+b_{1} s_{n}\left(t-\tau_{n}\right)+c_{1} s_{n+1}(t) \\
& +d_{1} s_{n+1}\left(t-\tau_{n}\right)+e_{1} s_{n+1}\left(t-\tau_{n}^{c}-\tau_{n}\right),
\end{aligned}
$$

where

$$
a_{1}=\left[\begin{array}{ccc}
-\frac{1}{T_{n}} & 0 & 0 \\
1 & 0 & 0 \\
0 & -1 & 0
\end{array}\right],
$$$$
b_{1}=\left[\begin{array}{ccc}
0 & -\frac{k_{n}^{\text {hum }}+\lambda_{n}^{\text {hum }}}{T_{n}} & \frac{k_{n}^{\text {hum }} \Lambda}{T_{n}} \\
0 & 0 & 0 \\
0 & 0 & 0
\end{array}\right] \text {, }
$$$$
c_{1}=\left[\begin{array}{lll}
0 & 0 & 0 \\
0 & 0 & 0 \\
0 & 1 & 0
\end{array}\right],
$$

$$
e_{1}=\left[\begin{array}{lll}
0 & 0 & 0 \\
0 & 0 & 0 \\
0 & 0 & 0
\end{array}\right] \text {. }
$$


Same as the manual driving scenario, the error dynamic for automated driving can be obtained as

$$
\begin{aligned}
\delta \dot{a}_{n}(t)= & \frac{k_{n}^{\mathrm{CACC}}}{T_{n}}\left[\Lambda \delta y_{n}\left(t-\tau_{n}\right)-\delta v_{n}\left(t-\tau_{n}\right)\right] \\
& +\frac{\lambda_{n}^{\mathrm{CACC}}}{T_{n}}\left(\delta v_{n+1}\left(t-\tau_{n}\right)-\delta v_{n}\left(t-\tau_{n}\right)\right) \\
& +\frac{\gamma_{n}^{\mathrm{CACC}}}{T_{n}} \delta a_{n+1}\left(t-\tau_{n}^{c}-\tau_{n}\right)-\frac{1}{T_{n}} \delta a_{n}(t) \\
\delta \dot{v}_{n}(t)= & \delta a_{n}(t) \\
\delta \dot{y}_{n}(t)= & \delta v_{n+1}(t)-\delta v_{n}(t) .
\end{aligned}
$$

Reformulate the error dynamic (8) as

$$
\begin{aligned}
\dot{s}_{n}(t)= & a_{2} s_{n}(t)+b_{2} s_{n}\left(t-\tau_{n}\right)+c_{2} s_{n+1}(t) \\
& +d_{2} s_{n+1}\left(t-\tau_{n}\right)+e_{2} s_{n+1}\left(t-\tau_{n}^{c}-\tau_{n}\right),
\end{aligned}
$$

where

$$
\begin{aligned}
& a_{2}=\left[\begin{array}{ccc}
-\frac{1}{T_{n}} & 0 & 0 \\
1 & 0 & 0 \\
0 & -1 & 0
\end{array}\right], \\
& b_{2}=\left[\begin{array}{ccc}
0 & -\frac{k_{n}^{\mathrm{CACC}}+\lambda_{n}^{\mathrm{CACC}}}{T_{n}} & \frac{k_{n}^{\mathrm{CACC}} \Lambda}{T_{n}} \\
0 & 0 & 0 \\
0 & & 0
\end{array}\right], \\
& c_{2}=\left[\begin{array}{lll}
0 & 0 & 0 \\
0 & 0 & 0 \\
0 & 1 & 0
\end{array}\right], \\
& d_{2}=\left[\begin{array}{lll}
0 & \\
0 & \frac{\lambda_{n}^{\mathrm{CACC}}}{T_{n}} & 0 \\
0 & 0 & 0 \\
0 & 0 & 0
\end{array}\right], \\
& e_{2}=\left[\begin{array}{lll}
\frac{\gamma_{n}^{\mathrm{CACC}}}{T_{n}} & 0 & 0 \\
0 & 0 & 0 \\
0 & 0 & 0
\end{array}\right] .
\end{aligned}
$$

Define the augmented vector $S(t)=\left[\begin{array}{lll}s_{N}(t) & s_{N-1}(t) & \cdots\end{array}\right.$ $\left.s_{1}(t)\right]^{T}$, which consists of the state variables of all vehicles in the platoon; according to (5)-(9), the state space expression of the cooperative platoon can be described as

$$
\begin{array}{r}
\dot{S}(t)=A_{1} S(t)+B_{1} S\left(t-\tau_{n}\right)+W_{1} S\left(t-\tau_{n}^{c}-\tau_{n}\right) \\
\quad \text { for manual driving } \\
\dot{S}(t)=A_{2} S(t)+B_{2} S\left(t-\tau_{n}\right)+W_{2} S\left(t-\tau_{n}^{c}-\tau_{n}\right)
\end{array}
$$

where

$$
A_{1}=\left[\begin{array}{ccccc}
a_{1} & 0 & 0 & \cdots & c_{1} \\
c_{1} & a_{1} & 0 & \cdots & 0 \\
0 & c_{1} & a_{1} & \cdots & 0 \\
\vdots & \vdots & \vdots & \ddots & \vdots \\
0 & \cdots & 0 & c_{1} & a_{1}
\end{array}\right],
$$$$
B_{1}=\left[\begin{array}{ccccc}
b_{1} & 0 & 0 & \cdots & d_{1} \\
d_{1} & b_{1} & 0 & \cdots & 0 \\
0 & d_{1} & b_{1} & \cdots & 0 \\
\vdots & \vdots & \vdots & \ddots & \vdots \\
0 & \cdots & 0 & d_{1} & b_{1}
\end{array}\right] \text {, }
$$$$
W_{1}=\left[\begin{array}{ccccc}
0 & 0 & 0 & \cdots & 0 \\
0 & 0 & 0 & \cdots & 0 \\
0 & 0 & 0 & \cdots & 0 \\
\vdots & \vdots & \vdots & \ddots & \vdots \\
0 & \cdots & 0 & 0 & 0
\end{array}\right],
$$$$
A_{2}=\left[\begin{array}{ccccc}
a_{2} & 0 & 0 & \cdots & c_{2} \\
c_{2} & a_{2} & 0 & \cdots & 0 \\
0 & c_{2} & a_{2} & \cdots & 0 \\
\vdots & \vdots & \vdots & \ddots & \vdots \\
0 & \cdots & 0 & c_{2} & a_{2}
\end{array}\right] \text {, }
$$

$$
\begin{gathered}
B_{2}=\left[\begin{array}{ccccc}
b_{2} & 0 & 0 & \cdots & d_{2} \\
d_{2} & b_{2} & 0 & \cdots & 0 \\
0 & d_{2} & b_{2} & \cdots & 0 \\
\vdots & \vdots & \vdots & \ddots & \vdots \\
0 & \cdots & 0 & d_{2} & b_{2}
\end{array}\right], \\
W_{2}=\left[\begin{array}{ccccc}
0 & 0 & 0 & \cdots & e_{2} \\
e_{2} & 0 & 0 & \cdots & 0 \\
0 & e_{2} & 0 & \cdots & 0 \\
\vdots & \vdots & \vdots & \ddots & \vdots \\
0 & \cdots & 0 & e_{2} & 0
\end{array}\right] .
\end{gathered}
$$

In order to keep drivers in the loop, which means drivers should always monitor the vehicle's running state and supervise the change of driving environment to an adequate level even if the longitudinal and/or lateral driving tasks were conducted by ADAS or automated driving system, the periodic switched driving strategy is put forth creatively to avoid driver fatigue or distraction which may cause serious traffic accidents and fateful consequences. From the perspective of 
control system, according to (11), the switched cooperative driving model can be regarded as a switched control system with varying time delay, which can be expressed as

$$
\begin{aligned}
\dot{S}(t)= & A_{\sigma\left(t_{k}\right)} S(t)+B_{\sigma\left(t_{k}\right)} S\left(t-\tau_{n}\right) \\
& +W_{\sigma\left(t_{k}\right)} S\left(t-\tau_{n}^{c}-\tau_{n}\right) \quad t \in\left[t_{k}, t_{k+1}\right), k \in \mathbb{N},
\end{aligned}
$$

where $\sigma(t):[0,+\infty) \rightarrow \bar{M}=\{1,2\}$ is a piecewise constant function called the switching signal which specifies the active subsystem at each time; only one subsystem is active at each sampling instant. For a switching sequence $0 \leq t_{0}<t_{1}<$ $\cdots<t_{k}<t_{k+1}<\cdots, \sigma(t)$ is everywhere continuous from the right; when $t \in\left[t_{k}, t_{k+1}\right)$, we say the $\sigma\left(t_{k}\right)$ th subsystem is active.

\section{Stability Analysis}

For all types of vehicle-to-vehicle cooperative driving systems, stability analysis is definitely an important issue. The existing stability analysis for the cooperative driving mainly focuses on the stability condition from the viewpoint of fluid mechanics. In fact, from the perspective of cyberphysical system, the cooperative driving system is a nonlinear and strong coupling complex system. So, it is meaningful to analyze the switched cooperative driving system from the control system point of view.

To obtain the main results, the following definition and lemmas are needed.

Definition 4. System (13) is said to be exponentially stable under switching signal $\sigma(t)$ if the solution of system (13) satisfies

$$
\|S(t)\|^{2} \leq \kappa e^{-\beta\left(t-t_{0}\right)}\left\|S\left(t_{0}\right)\right\|_{c 1}^{2}, \quad \forall t \geq 0,
$$

for $\kappa \geq 1$ and $\beta>0$, where $\|S(t)\|_{c 1}=\sup _{-\tau_{n}^{c}-\tau_{n} \leq \theta \leq 0}\{\| S(t+$ $\theta)\|,\| \dot{S}(t+\theta) \|\}$.

Lemma 5. The matrix inequality

$$
\left[\begin{array}{cc}
S_{11} & S_{12} \\
S_{12}^{T} & -S_{22}
\end{array}\right]<0
$$

where $S_{11}=S_{11}^{T}, S_{22}=S_{22}^{T}$ are equivalent to $S_{22}>0, S_{11}+$ $S_{12} S_{22}^{-1} S_{12}^{T}<0$ and $S_{11}<0, S_{22}+S_{12}^{T} S_{11}^{-1} S_{12}>0$.

Lemma 6. For any constant matrix $Z>0$, scalar $\tau_{2}>\tau_{1} \geq 0$, $\alpha \neq 0$, and vector function $x:\left[t-\tau_{2}, t-\tau_{1}\right] \rightarrow \mathbb{R}^{n}$, one has

$$
\begin{gathered}
\frac{e^{\alpha \tau_{2}}-e^{\alpha \tau_{1}}}{\alpha} \int_{t-\tau_{2}}^{t-\tau_{1}} e^{\alpha(s-t)} x^{T}(s) Z x(s) d s \\
\geq \int_{t-\tau_{2}}^{t-\tau_{1}} x^{T}(s) d s Z \int_{t-\tau_{2}}^{t-\tau_{1}} x(s) d s .
\end{gathered}
$$

Proof. According to the Schur complement theorem, together with $Z>0$, it holds that

$$
\begin{aligned}
{\left[\begin{array}{cc}
e^{\alpha(s-t)} x^{T}(s) Z x(s) & x^{T}(s) \\
x(s) & e^{\alpha(t-s)}
\end{array}\right] \geq 0, } & \\
& t-\tau_{2} \leq s \leq t-\tau_{1} ;
\end{aligned}
$$

taking the integral of formula (17) over the closed interval $[t-$ $\left.\tau_{2}, t-\tau_{1}\right]$ yields

$$
\left[\begin{array}{ccc}
\int_{t-\tau_{2}}^{t-\tau_{1}} e^{\alpha(s-t)} x^{T}(s) Z x(s) d s & \int_{t-\tau_{2}}^{t-\tau_{1}} x^{T}(s) d s \\
& \int_{t-\tau_{2}}^{t-\tau_{1}} x(s) d s & \frac{e^{\alpha \tau_{2}}-e^{\alpha \tau_{1}}}{\alpha} Z^{-1}
\end{array}\right] \geq 0 .
$$

Then, by Schur complement theorem, one can get that the matrix inequality (18) is equivalent to (16). The proof of Lemma 6 is complete.

In this paper, we use $P>0(\geq,<, \leq 0)$ to denote a positive definite (semidefinite, negative definite, and seminegative definite) matrix $P . \lambda_{\max }(P)$ and $\lambda_{\min }(P)$ denote the maximum and minimum eigenvalues of $P$. $I$ and 0 represent identity matrix and zero matrix in the block matrix. The superscript " $T$ " stands for matrix transpose, and the symmetric terms in a matrix are denoted by $*$.

Without loss of generality, we regard the cooperative system with manual driving considering the vehicle dynamics as an unstabilizable system according to the idea of stability analysis from [31,32], when the drivers' sensitivity coefficient is low. In the research about the platoon of vehicles, the authors of [3] suggest that CACC can guarantee the string stability of the cooperative driving system, which means the effect of disturbances is attenuated along the vehicle string, due to the fact that vehicles can obtain the control information of preceding vehicles beforehand via intervehicle communication before the change of headway and velocity difference. So, the cooperative system with automated driving can be considered as a stabilizable system.

In the following, for the sake of convenience, suppose that $M^{-}$corresponds to the stable subsystems of (13) and $M^{+}$corresponds to the unstable subsystems. Obviously, $M^{-} \mathrm{U}$ $M^{+}=\bar{M}$.

It should be noted that once the unstable subsystem is active in a hybrid system, the stability of the whole system usually cannot be guaranteed. However, if the total activation time of the unstable subsystems is relatively small compared with the stable subsystems, the stability of the system can be achieved.

Theorem 7. For given positive constants $\alpha_{1}, \alpha_{2}, \tau_{n}$, and $\tau_{n}^{c}$, if there exist symmetric positive definite matrices $P_{k}, Q_{i k}, R_{i k}, i \in$ $(1,2), k \in(1,2, \ldots, p)$, such that

$$
\Phi^{k}=\left[\begin{array}{ccc}
\Phi_{11}^{k} & \Phi_{12}^{k} & \Phi_{13}^{k} \\
* & \Phi_{22}^{k} & \Phi_{23}^{k} \\
* & * & \Phi_{33}^{k}
\end{array}\right]<0
$$


holds, for the Lyapunov-Krasovskii function candidate

$$
\begin{aligned}
V_{k}(t)= & S^{T}(t) P_{k} S(t) \\
& +\int_{t-\tau_{n}}^{t} e^{\alpha_{k}(u-t)} S^{T}(u) Q_{1 k} S(u) d u \\
& +\int_{t-\tau_{n}^{c}-\tau_{n}}^{t} e^{\alpha_{k}(u-t)} S^{T}(u) Q_{2 k} S(u) d u \\
& +\int_{-\tau_{n}}^{0} \int_{t+\theta}^{t} e^{\alpha_{k}(u-t)} \dot{S}^{T}(u) R_{1 k} \dot{S}(u) d u d \theta \\
& +\int_{-\tau_{n}^{c}-\tau_{n}}^{0} \int_{t+\theta}^{t} e^{\alpha_{k}(u-t)} \dot{S}^{T}(u) R_{2 k} \dot{S}(u) d u d \theta
\end{aligned}
$$

along the trajectory of system (13), there holds the following inequality:

$$
V_{k}(t) \leq e^{-\alpha_{k}\left(t-t_{k}\right)} V_{k}\left(t_{k}\right)
$$

where

$$
\begin{aligned}
\Phi_{11}^{k}= & A_{\sigma\left(t_{k}\right)}^{T} P_{k}+P_{k} A_{\sigma\left(t_{k}\right)}+\alpha_{k} P_{k}+Q_{1 k}+Q_{2 k} \\
& +\tau_{n} R_{1 k}+\left(\tau_{n}^{c}+\tau_{n}\right) R_{2 k}+\frac{\alpha_{k}}{1-e^{\alpha_{k} \tau_{n}}} R_{1 k} \\
& +\frac{\alpha_{k}}{1-e^{\alpha_{k}\left(\tau_{n}^{c}+\tau_{n}\right)}} R_{2 k}, \\
\Phi_{12}^{k}= & P_{k} B_{\sigma\left(t_{k}\right)}+\frac{\alpha_{k}}{e^{\alpha_{k} \tau_{n}}-1} R_{1 k}+\frac{\alpha_{k}}{e^{\alpha_{k}\left(\tau_{n}^{c}+\tau_{n}\right)}-1} R_{2 k}, \\
\Phi_{13}^{k}= & P_{k} W_{\sigma\left(t_{k}\right)}, \\
\Phi_{22}^{k}= & -e^{\alpha_{k} \tau_{n}} Q_{1 k}+\frac{\alpha_{k}}{1-e^{\alpha_{k} \tau_{n}}} R_{1 k}+\frac{\alpha_{k}}{1-e^{\alpha_{k}\left(\tau_{n}^{c}+\tau_{n}\right)}} R_{2 k}, \\
\Phi_{23}^{k}= & 0, \\
\Phi_{33}^{k}= & -e^{\alpha_{k}\left(\tau_{n}^{c}+\tau_{n}\right)} Q_{2 k}, \\
\alpha_{k}= & \begin{cases}\alpha_{1} \quad k \in M^{-} \\
\alpha_{2} \quad k \in M^{+} .\end{cases}
\end{aligned}
$$

Proof. Suppose that system (13) is in the $k$ th mode for $t \in\left[t_{k}, t_{k+1}\right)$; one can construct the Lyapunov-Krasovskii function candidate for the switched cooperative driving model (13) as (20).

Taking the derivative of $V_{k}(t)$ along the trajectory of model (13) yields

$$
\begin{aligned}
& \dot{V}_{k}(t) \\
& =2 S^{T}(t) P_{k} \dot{S}(t)+S^{T}(u) Q_{1 k} S(u) \\
& \quad-e^{-\alpha_{k} \tau_{n}} S^{T}\left(t-\tau_{n}\right) Q_{1 k} S\left(t-\tau_{n}\right) \\
& \quad-\alpha_{k} \int_{t-\tau_{n}}^{t} e^{\alpha_{k}(u-t)} S^{T}(u) Q_{1 k} S(u) d u \\
& \quad+S^{T}(t) Q_{2 k} S(t)
\end{aligned}
$$

$$
\begin{aligned}
& -e^{-\alpha_{k}\left(\tau_{n}^{c}+\tau_{n}\right)} S^{T}\left(t-\tau_{n}^{c}-\tau_{n}\right) Q_{2 k} S\left(t-\tau_{n}^{c}-\tau_{n}\right) \\
& -\alpha_{k} \int_{t-\tau_{n}^{c}-\tau_{n}}^{t} e^{\alpha_{k}(u-t)} S^{T}(u) Q_{2 k} S(u) d u \\
& +\tau_{n} \dot{S}^{T}(t) R_{1 k} \dot{S}(t)+\left(\tau_{n}^{c}+\tau_{n}\right) \dot{S}^{T}(t) R_{2 k} \dot{S}(t) \\
& -\int_{t-\tau_{n}}^{t} e^{\alpha_{k}(u-t)} \dot{S}^{T}(u) R_{1 k} \dot{S}(u) d u \\
& -\alpha_{k} \int_{-\tau_{n}}^{0} \int_{t+\theta}^{t} e^{\alpha_{k}(u-t)} \dot{S}^{T}(u) R_{1 k} \dot{S}(u) d u d \theta \\
& -\int_{t-\tau_{n}^{c}-\tau_{n}}^{t} e^{\alpha_{k}(u-t)} \dot{S}^{T}(u) R_{2 k} \dot{S}(u) d u \\
& -\alpha_{k} \int_{-\tau_{n}}^{0} \int_{t+\theta}^{t} e^{\alpha_{k}(u-t)} \dot{S}^{T}(u) R_{2 k} \dot{S}(u) d u d \theta .
\end{aligned}
$$

Then, direct computation gives

$$
\begin{aligned}
\dot{V}_{k}(t) & +\alpha_{k} V_{k}(t) \\
= & 2 S^{T}(t) P_{k} \dot{S}(t)+\alpha_{k} S^{T}(t) P_{k} S(t) \\
& +S^{T}(u) Q_{1 k} S(u) \\
& -e^{-\alpha_{k} \tau_{n}} S^{T}\left(t-\tau_{n}\right) Q_{1 k} S\left(t-\tau_{n}\right) \\
& +S^{T}(t) Q_{2 k} S(t) \\
& -e^{-\alpha_{k}\left(\tau_{n}^{c}+\tau_{n}\right)} S^{T}\left(t-\tau_{n}^{c}-\tau_{n}\right) Q_{2 k} S\left(t-\tau_{n}^{c}-\tau_{n}\right) \\
& +\tau_{n} \dot{S}^{T}(t) R_{1 k} \dot{S}^{(t)} \\
& -\int_{t-\tau_{n}}^{t} e^{\alpha_{k}(u-t)} \dot{S}^{T}(u) R_{1 k} \dot{S}(u) d u \\
& +\left(\tau_{n}^{c}+\tau_{n}\right) \dot{S}^{T}(t) R_{2 k} \dot{S}(t) \\
& -\int_{t-\tau_{n}^{c}-\tau_{n}}^{t} e^{\alpha_{k}(u-t)} \dot{S}^{T}(u) R_{2 k} \dot{S}(u) d u .
\end{aligned}
$$

In addition, by Lemma 6 , it holds that

$$
\begin{aligned}
& -\int_{t-\tau_{n}}^{t} e^{\alpha_{k}(u-t)} \dot{S}^{T}(u) R_{1 k} \dot{S}(u) d u \\
& \quad \leq \frac{\alpha_{k}}{1-e^{\alpha_{k} \tau_{n}}}\left(S^{T}(t)-S^{T}\left(t-\tau_{n}\right)\right) \\
& \cdot R_{1 k}\left(S(t)-S\left(t-\tau_{n}\right)\right), \\
& -\int_{t-\tau_{n}^{c}-\tau_{n}}^{t} e^{\alpha_{k}(u-t)} \dot{S}^{T}(u) R_{2 k} \dot{S}(u) d u \\
& \leq \frac{\alpha_{k}}{1-e^{\alpha_{k}\left(\tau_{n}^{c}+\tau_{n}\right)}}\left(S^{T}(t)-S^{T}\left(t-\tau_{n}^{c}-\tau_{n}\right)\right) \\
& \cdot R_{2 k}\left(S(t)-S\left(t-\tau_{n}^{c}-\tau_{n}\right)\right) .
\end{aligned}
$$


Combining (24)-(25) yields

$$
\begin{aligned}
& \dot{V}_{k}(t)+\alpha_{k} V_{k}(t) \leq 2 S^{T}(t) P_{k} \dot{S}(t)+\alpha_{k} S^{T}(t) P_{k} S(t) \\
& +S^{T}(u) Q_{1 k} S(u)-e^{-\alpha_{k} \tau_{n}} S^{T}\left(t-\tau_{n}\right) Q_{1 k} S\left(t-\tau_{n}\right) \\
& +S^{T}(t) Q_{2 k} S(t)-e^{-\alpha_{k}\left(\tau_{n}^{c}+\tau_{n}\right)} S^{T}\left(t-\tau_{n}^{c}-\tau_{n}\right) \\
& \cdot Q_{2 k} S\left(t-\tau_{n}^{c}-\tau_{n}\right)+\tau_{n} \dot{S}^{T}(t) R_{1 k} \dot{S}(t) \\
& +\frac{\alpha_{k}}{1-e^{\alpha_{k} \tau_{n}}}\left(S^{T}(t)-S^{T}\left(t-\tau_{n}\right)\right) \\
& \cdot R_{1 k}\left(S(t)-S\left(t-\tau_{n}\right)\right)+\left(\tau_{n}^{c}+\tau_{n}\right) \dot{S}^{T}(t) R_{2 k} \dot{S}(t) \\
& +\frac{\alpha_{k}}{1-e^{\alpha_{k}\left(\tau_{n}^{c}+\tau_{n}\right)}}\left(S^{T}(t)-S^{T}\left(t-\tau_{n}^{c}-\tau_{n}\right)\right) \\
& \cdot R_{2 k}\left(S(t)-S\left(t-\tau_{n}^{c}-\tau_{n}\right)\right) .
\end{aligned}
$$

Let $\xi(t)=\left[S(t), S\left(t-\tau_{n}\right), S\left(t-\tau_{n}-\tau_{n}^{c}\right)\right]^{T}$; inequality (26) can be rewritten as

$$
\dot{V}_{k}(t)+\alpha_{k} V_{k}(t) \leq \xi(t)^{T} \Phi^{k} \xi(t) .
$$

If (19) holds, one has

$$
\dot{V}_{k}(t)+\alpha_{k} V_{k}(t) \leq 0 .
$$

Integrating inequality (28) from $t_{k}$ to $t$ gives $V_{k}(t) \leq$ $e^{-\alpha_{k}\left(t-t_{k}\right)} V_{k}\left(t_{k}\right)$; the proof of Theorem 7 is complete.

\section{Design of the Switching Signal}

Now, for any piecewise constant switching signal $\sigma(t)$ and any $0 \leq t_{0}<t$, we let $T^{-}\left(t_{0}, t\right)$ (resp., $T^{+}\left(t_{0}, t\right)$ ) denote the total activation time of stabilizable (resp., unstabilizable) subsystems during $\left(t_{0}, t\right)$. $N^{-}$represents the total number of system switching instances from stabilizable subsystems to unstabilizable subsystems during $\left(t_{0}, t\right)$; conversely, $N^{+}$is the number of system switching instances from unstabilizable subsystems to stabilizable subsystems.

Theorem 8. For given positive constants $\alpha_{1}, \alpha_{2}, \mu_{1}, \mu_{2}, \tau_{n}$, and $\tau_{n}^{c}$, if there exist symmetric positive definite matrices $P_{k}, Q_{i k}, R_{i k}, i \in(1,2), k \in(1,2, \ldots, p)$, such that Theorem 7 and inequalities

$$
\begin{gathered}
P_{k} \leq \mu_{1} P_{l}, \\
Q_{i k} \leq \mu_{1} Q_{i l}, \\
R_{i k} \leq \mu_{1} R_{i l},
\end{gathered}
$$

$\forall k, l \in M$

hold, where $\mu_{1} \geq 1$, system (13) is exponentially stable for any switching signal satisfying the following condition:

$$
\begin{aligned}
\frac{T^{-}}{T^{+}} & \geq \frac{\left(\alpha_{2}+\alpha^{*}\right)}{\left(\alpha_{1}-\alpha^{*}\right)}, \quad \alpha^{*} \in\left(0, \alpha_{1}\right) \\
f\left(t_{0}, t\right) & \leq \frac{\alpha}{\ln \left(\mu_{1} \mu_{2}\right)}, \quad \alpha \in\left(0, \alpha^{*}\right),
\end{aligned}
$$

where $f\left(T_{1}, T_{2}\right)=N^{+} /\left(T_{2}-T_{1}\right), \mu_{2}=e^{\left(\alpha_{1}+\alpha_{2}\right)\left(\tau_{n}^{c}+\tau_{n}\right)} \mu_{1}$.
Proof. According to Theorem 7, we have

$$
V_{k}(t) \leq \begin{cases}e^{-\alpha_{1}\left(t-t_{k}\right)} V_{k}\left(t_{k}\right), & k \in M^{-} \\ e^{\alpha_{2}\left(t-t_{k}\right)} V_{k}\left(t_{k}\right), & k \in M^{+} .\end{cases}
$$

At switching time $t_{k}$, based on (20) and (29), one has

$$
V_{k}\left(t_{k}^{+}\right) \leq \begin{cases}\mu_{1} V_{p}\left(t_{k}^{-}\right), & k \in M^{-}, p \in M^{+}, \\ \mu_{2} V_{p}\left(t_{k}^{-}\right), & k \in M^{+}, p \in M^{-},\end{cases}
$$

where $\mu_{2}=e^{\left(\alpha_{1}+\alpha_{2}\right)\left(\tau_{n}^{c}+\tau_{n}\right)} \mu_{1}$.

Without loss of generality, when $t \in\left[t_{k}, t_{k+1}\right), k \in M^{+}$, combining (31)-(32), along the trajectory of system (13), yields

$$
V(t) \leq \mu_{1}^{N^{-}} \mu_{2}^{N^{+}} e^{-\alpha_{1} T^{-}\left(t_{0}, t\right)+\alpha_{2} T^{+}\left(t_{0}, t\right)} V\left(t_{0}\right) .
$$

By (20), it holds that

$$
\begin{gathered}
V(t) \geq a\|S(t)\|^{2}, \\
V\left(t_{0}\right) \leq b\left\|S\left(t_{0}\right)\right\|_{c 1}^{2} .
\end{gathered}
$$

Because of $N^{-}\left(t_{0}, t\right) \leq N^{+}\left(t_{0}, t\right)$,

$$
\|S(t)\|^{2} \leq \frac{1}{a} V(t) \leq \frac{b}{a} e^{-\left(\alpha^{*}-\alpha\right)\left(t-t_{0}\right)}\left\|S\left(t_{0}\right)\right\|_{c 1}^{2} .
$$

According to Definition 4, system (13) is exponentially stable when (30) holds, where

$$
\begin{aligned}
& a= \min _{\forall k \in M} \lambda\left(P_{k}\right), \\
& b=\max _{\forall k \in M} \lambda\left(P_{k}\right)+\tau_{n} \max _{\forall k \in M} \lambda\left(Q_{1 k}\right) \\
&+\left(\tau_{n}+\tau_{n}^{c}\right) \max _{\forall k \in M} \lambda\left(Q_{2 k}\right) \\
&+\frac{1}{2}\left(\tau_{n}\right)^{2} \max _{\forall k \in M} \lambda\left(R_{1 k}\right) \\
&+\frac{1}{2}\left(\tau_{n}+\tau_{n}^{c}\right)^{2} \max _{\forall k \in M} \lambda\left(R_{2 k}\right) .
\end{aligned}
$$

The proof of Theorem 8 is complete.

Theorem 8 provides a sufficient condition to choose a proper switching signal such that the switched cooperative driving system (13) is stable even in the existence of a certain amount of communication delay. The matrix $P_{k}, Q_{i k}, R_{i k}, i \in$ $(1,2), k \in(1,2, \ldots, p)$, reduces the conservativeness of the proposed method. Additionally, when $\alpha_{1}, \alpha_{2}, \mu_{1}, \mu_{2}, \tau_{n}$, and $\tau_{n}^{c}$ are given, it is obvious that the condition in Theorems 7 and 8 takes the form of linear matrix inequality, which can be easily determined using the Matlab LMI toolbox.

Reference [7] investigated the driver behavior when resuming control from a highly automated vehicle; the study suggests that drivers require around $30-40 \mathrm{~s}$ to resume adequate and stable control of driving from automation. So, single manual driving time should not be less than $30 \mathrm{~s}$ when designing the switching signal for the sake of safety. 


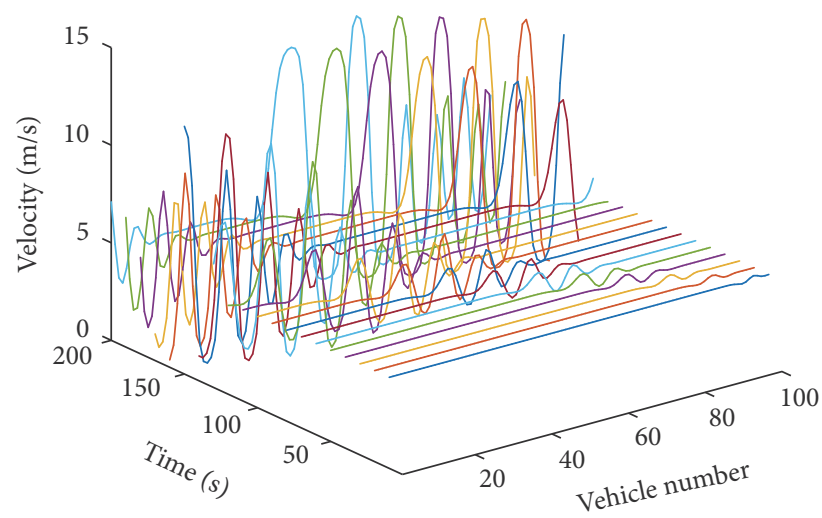

FIGURE 2: Velocity variation of all vehicles for manual driving.

Furthermore, the suggested switching period should not exceed $6 \mathrm{~min}$ to keep drivers in the loop according to the previous works about human factors.

According to these conditions and suggestions, a proper switching signal can be easily designed using the Matlab LMI toolbox.

\section{Numerical Simulation}

In this section, numerical examples are given to illustrate the effectiveness of the proposed methods for the switched cooperative driving system towards human vehicle copiloting situation considering the vehicle dynamic and realistic intervehicle communication.

Let us consider a case where 100 vehicles are running on a single lane under a periodic boundary condition, with the length $L=1500 \mathrm{~m}$. We set the initial disturbance with reference to [32]. The simulation is conducted for $2000 \mathrm{~s}$ with the evolution of time. $x_{1}(0)=1 \mathrm{~m}, x_{n}(0)=(n-1) L / N \mathrm{~m}$, for $i=2, \ldots, 100, v_{n}(0)=V(L / N)$, for $i=1, \ldots, 100$.

In manual driving situation, the driver's sensitivity is $k_{n}^{\text {hum }}=0.41$, and the sensitivity coefficient of the velocity difference is $\lambda_{n}^{\text {hum }}=0.5$. Assuming that $T_{n}=0.2$ and $\tau_{n}=$ $0.2 \mathrm{~s}$, Figure 2 plots the velocity variations of all vehicles, and Figure 3 shows the snapshots of the velocity of all vehicles at different times.

In Figure 2, the three axes represent the number of the vehicles, the time of the evolution, and the velocity of the vehicles, respectively. This figure depicts the spacetime evolution of the platoon when the 1st vehicle suffers an unexpected speed reduction. From this figure, we can see that the speed fluctuation will become severe along the platoon sequence and the initial uniform traffic flow will evolve into a propagating backward traffic congestion under the disturbance.

To further exhibit the effect of the unexpected speed reduction, the velocity profiles were obtained by taking snapshots of the evolution depicted by Figure 2 at time steps $t=10 \mathrm{~s}, 300 \mathrm{~s}$, and $2000 \mathrm{~s}$, respectively, as Figure 3. Figure 3 shows that many vehicles have to decrease their velocities to zero, which means a severe traffic jam occurs at time step $t=2000 \mathrm{~s}$. This reveals that the platoon is unstable under

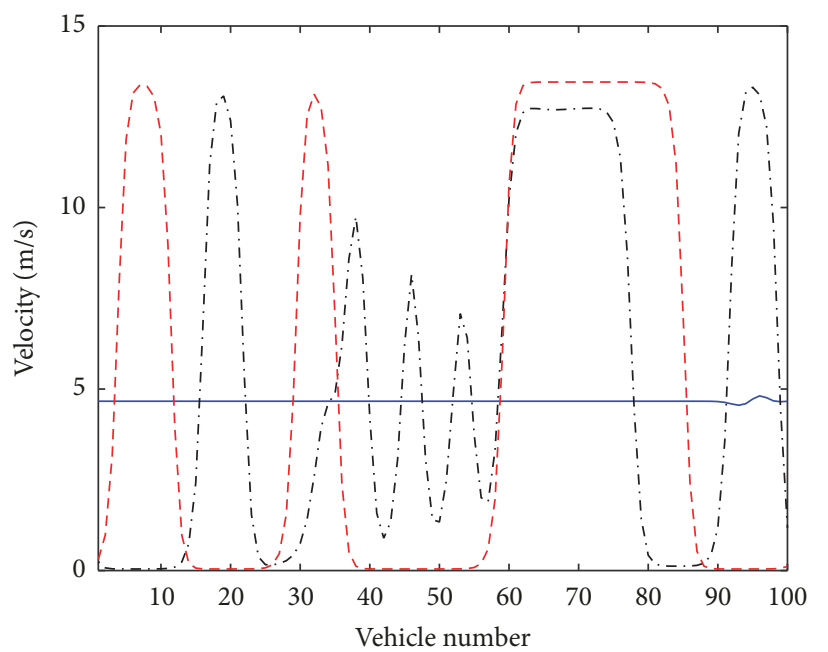

$$
\begin{aligned}
-t & =10 \mathrm{~s} \\
--t & =300 \mathrm{~s} \\
--t & =2000 \mathrm{~s}
\end{aligned}
$$

FIgURE 3: The snapshots of the velocity of all vehicles at different times for manual driving.

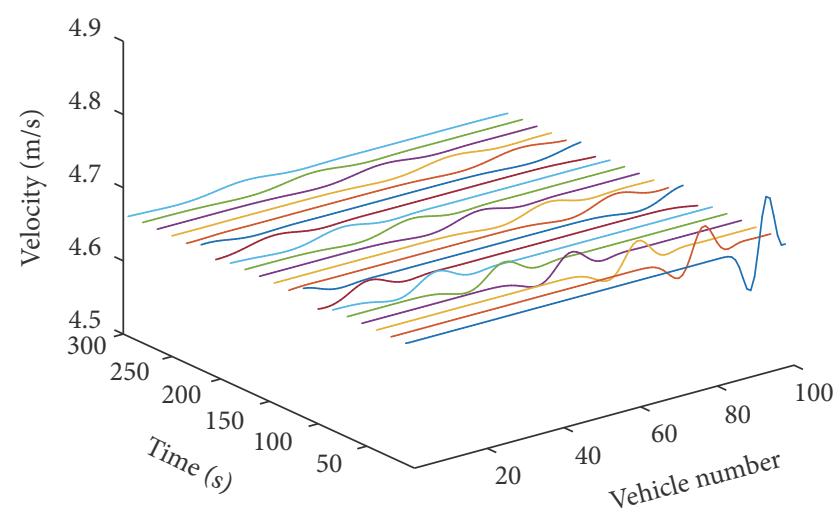

FIGURE 4: Velocity variation of all vehicles for automated driving.

manual driving which is suitable to the practice and the result of the traffic flow research.

Similarly, we set $k_{n}^{\mathrm{CACC}}=0.41, \lambda_{n}^{\mathrm{CACC}}=0.5$, and $\gamma_{n}^{\mathrm{CACC}}=$ 0.3 for the automated driving situation, when $T_{n}=0.2, \tau_{n}=$ $0.2 \mathrm{~s}$, and $\tau_{n}^{c}=0.2 \mathrm{~s}$; the simulations are shown in Figures 4 and 5 .

In contrast to Figures 2 and 3, we can see clearly from Figures 4 and 5 that the amplitudes of the velocity waves shrink distinctly when intervehicle wireless communication is taken into account and traffic flow becomes much smoother. This reveals that the CACC system can stabilize traffic flow and suppress traffic congestion efficiently, which is consistent with the studies on automated driving system.

From Figures $2-5$, it can be seen that the velocity fluctuations will decrease gradually as the simulation time develops under automated driving situation, even if there exists a certain amount of communication delay. However, the platoon is unstable and a traffic jam will occur under the manual driving case. These are in good agreement with the 


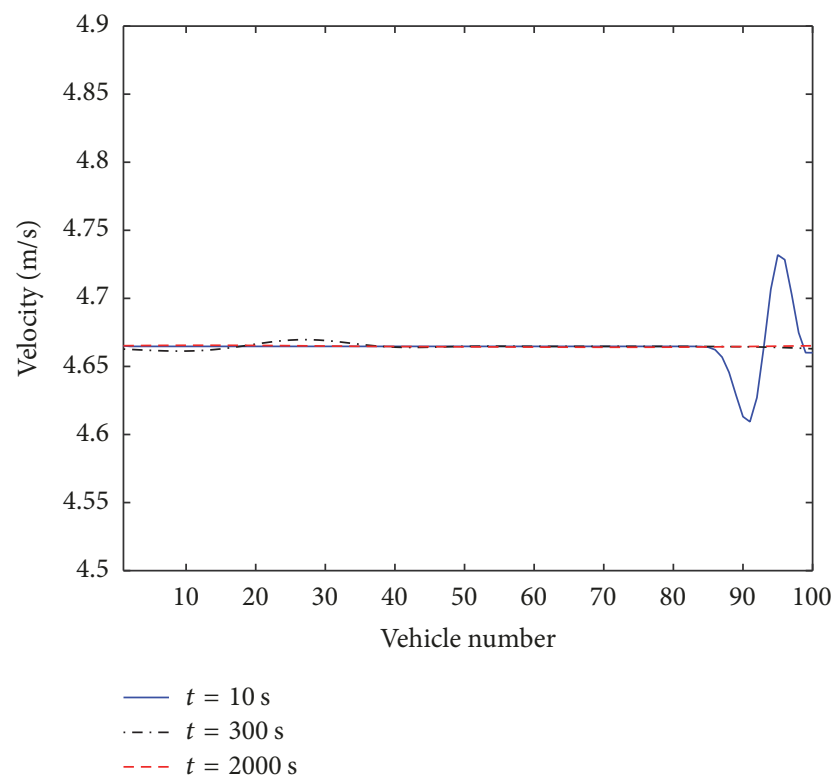

FIgURE 5: The snapshots of the velocity of all vehicles at different times for automated driving.

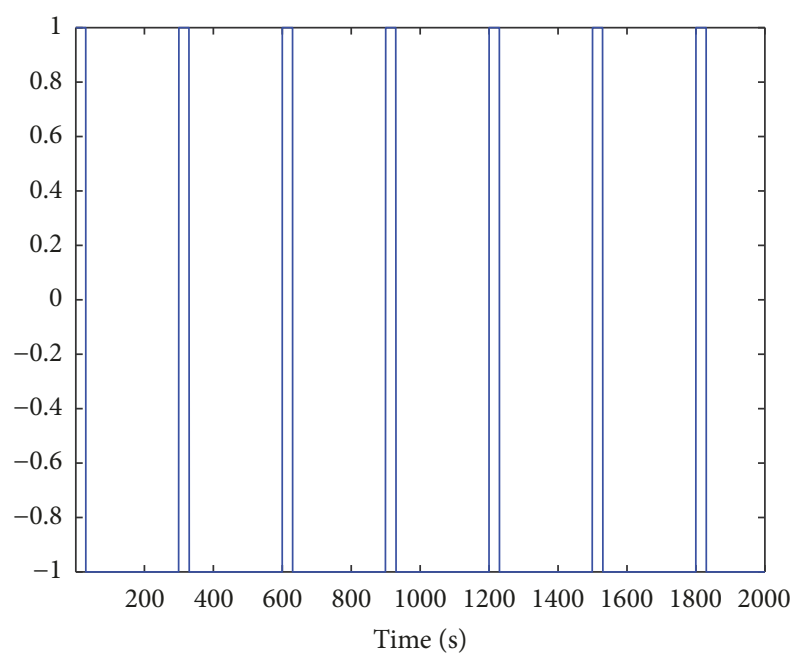

Figure 6: Switching signal.

aforementioned theoretical analysis, which can demonstrate indirectly that the constructed manual driving model and automated driving model are reasonable to a certain degree.

To demonstrate the switched cooperative driving model, according to Theorems 7 and 8, we can choose $\alpha_{1}=8$, $\alpha_{2}=2$, and $\mu_{1}=5$ using LMI toolbox, so the switching signal which satisfies condition (30) can be obtained as Figure 6 by selecting the adequate $\alpha^{*}$ and $\alpha$; the cycle length is $T=$ $300 \mathrm{~s}$, and the duty cycle is Duty $=0.1$, in which amplitude equal to one means the manual driving mode is active and amplitude equal to -1 means the current mode is automated driving. With the same initial condition and unexpected speed reduction of the 1st vehicle, the platoon revolution under the switching signal depicted by Figure 6 is shown in Figures 7 and 8.

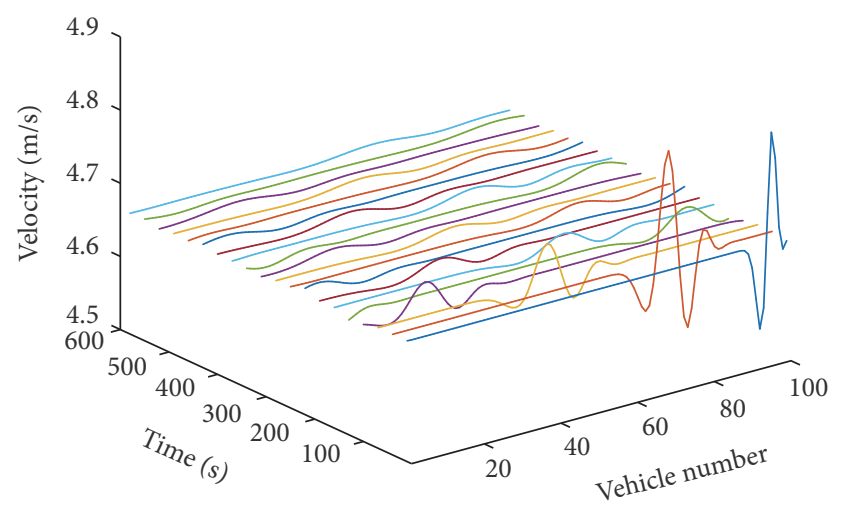

FIGURE 7: Velocity variation of all vehicles for switched cooperative driving model.

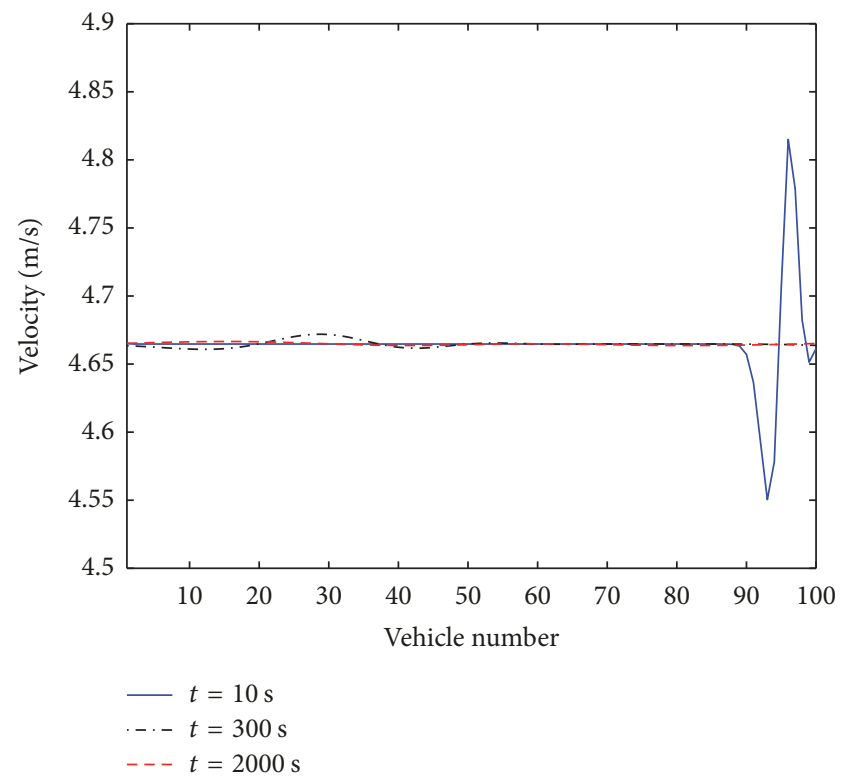

FIGURE 8: The snapshots of the velocity of all vehicles at different times for switched cooperative driving model.

Compared with Figures 4 and 5, we can see clearly from Figures 7 and 8 that the disturbance can also be well suppressed under the switching signal depicted by Figure 6, although the performance of the traffic flow declines slightly due to the periodic transitions between automated driving and manual driving. Fortunately, we can benefit significantly from the periodic switched driving strategy, by which drivers can be kept in the loop to respond to the takeover requests rapidly in L3 situation. Meanwhile, drivers' boredom, distraction, and fatigue caused by long-played supervising task can be prevented in L2 situation. Therefore, we can conclude that the numerical simulation is in good agreement with the analytical results.

\section{Conclusion}

In order to address the out-of-the-loop problem and distraction problem mentioned in the Introduction, this paper puts 
forth a periodic switched driving strategy to keep drivers always in the loop and prevent drivers' boredom, distraction, and even fatigue resulting from the supervising task. The potential impact of the transitions between automated and manual driving on traffic operations is revealed using the Lyapunov stability theory based on the proposed switched cooperative driving model towards the human vehicle copiloting situation. The switching signal with the comprehensive consideration of platoon stability and human factors can be easily designed based on the method presented by this paper. Numerical simulations illustrate the effectiveness of the proposed methods. The research results can provide scientific support for the optimization design of human vehicle copiloting.

\section{Conflicts of Interest}

The authors declare that there are no conflicts of interest regarding the publication of this paper.

\section{Acknowledgments}

This work was supported by the National Key R\&D Program (Grant no. 2016YFB0100904), the National Natural Science Foundation of China (Grant no. 61573075), the Natural Science Foundation of Chongqing (Grant no. cstc2017jcyjBX0001), and the Project of Standardization and New Model for Intelligent Manufacture (Grant no. 2016ZXFB06002).

\section{References}

[1] P. Varaiya and S. E. Shladover, "Sketch of an IVHS systems architecture," in Proceedings of the IEEE Vehicle Navigation and Information Systems Conference, VNISC '91, vol. 2, pp. 909-922, 1991.

[2] M. Wang, W. Daamen, S. P. Hoogendoorn, and B. van Arem, "Rolling horizon control framework for driver assistance systems. Part I: mathematical formulation and non-cooperative systems," Transportation Research Part C: Emerging Technologies, vol. 40, pp. 271-289, 2014.

[3] S. Lam, J. Taghia, and J. Katupitiya, "Evaluation of a transportation system employing autonomous vehicles," Journal of Advanced Transportation, vol. 50, no. 8, pp. 2266-2287, 2016.

[4] SAE On-Road Automated Vehicle Standards Committee, "Taxonomy and definitions for terms related to on-road motor vehicle automated driving systems," SAE Standard J3016, pp. 1$16,2014$.

[5] N. H. T. S. Administration, Preliminary statement of policy concerning automated vehicles, pp. 1-14, 2013.

[6] T. M. Gasser and D. Westhoff, "BASt-study: definitions of automation and legal issues in Germany," in Proceedings of the Road Vehicle Automation Workshop, 2012.

[7] N. Merat, A. H. Jamson, F. C. H. Lai, M. Daly, and O. M. J. Carsten, "Transition to manual: driver behaviour when resuming control from a highly automated vehicle," Transportation Research Part F: Traffic Psychology and Behaviour, vol. 27, pp. 274-282, 2014.
[8] N. A. Stanton, M. S. Young, and G. H. Walker, "The psychology of driving automation: a discussion with Professor Don Norman," International Journal of Vehicle Design, vol. 45, no. 3, pp. 289-306, 2007.

[9] M. R. Endsley and E. O. Kiris, "The out-of-the-loop performance problem and level of control in automation," Human Factors: The Journal of the Human Factors and Ergonomics Society, vol. 37, no. 2, pp. 381-394, 1995.

[10] D. B. Kaber and M. R. Endsley, "Out-of-the-loop performance problems and the use of intermediate levels of automation for improved control system functioning and safety," Process Safety Progress, vol. 16, no. 3, pp. 126-131, 1997.

[11] R. Parasuraman and V. Riley, "Humans and automation: use, misuse, disuse, abuse," Human Factors: The Journal of the Human Factors and Ergonomics Society, vol. 39, no. 2, pp. 230 253, 1997.

[12] P. A. Hancock, R. J. Jagacinski, R. Parasuraman, C. D. Wickens, G. F. Wilson, and D. B. Kaber, "Human-automation interaction research: past, present, and future," Ergonomics in Design, vol. 21, no. 2, pp. 9-14, 2013.

[13] J. C. F. De Winter, R. Happee, M. H. Martens, and N. A. Stanton, "Effects of adaptive cruise control and highly automated driving on workload and situation awareness: a review of the empirical evidence," Transportation Research Part F: Traffic Psychology and Behaviour, vol. 27, pp. 196-217, 2014.

[14] N. A. Stanton and M. S. Young, "Vehicle automation and driving performance," Ergonomics, vol. 41, no. 7, pp. 1014-1028, 1998.

[15] N. Merat and J. D. Lee, "Preface to the special section on human factors and automation in vehicles: designing highly automated vehicles with the driver in mind," Human Factors: The Journal of the Human Factors and Ergonomics Society, vol. 54, no. 5, pp. 681-686, 2012.

[16] Z. J. Lu and J. C. F. de Winter, "A review and framework of control authority transitions in automated driving," in Proceedings of the 6th International Conference on Applied Human Factors and Ergonomics (Ahfe '15) and the Affiliated Conferences, pp. 2510-2517, 2015.

[17] B. D. Seppelt, M. N. Lees, and J. D. Lee, "Driver distraction and reliance: adaptive cruise control in the context of sensor reliability and algorithm limits," in Proceedings of the $3 r d$ International Driving Symposium on Human Factors in Driver Assessment, Training and Vehicle Design, pp. 255-261, 2005.

[18] A. F. L. Larsson, K. Kircher, and J. A. Hultgren, "Learning from experience: familiarity with ACC and responding to a cut-in situation in automated driving," Transportation Research Part F: Traffic Psychology and Behaviour, vol. 27, pp. 229-237, 2014.

[19] G. F. B. Piccinini, C. M. Rodrigues, M. Leitão, and A. Simões, "Reaction to a critical situation during driving with Adaptive Cruise Control for users and non-users of the system," Safety Science, vol. 72, pp. 116-126, 2015.

[20] K. Kircher, A. Larsson, and J. A. Hultgren, "Tactical driving behavior with different levels of automation," IEEE Transactions on Intelligent Transportation Systems, vol. 15, no. 1, pp. 158-167, 2014.

[21] B. D. Seppelt and J. D. Lee, "Making adaptive cruise control (ACC) limits visible," International Journal of Human-Computer Studies, vol. 65, no. 3, pp. 192-205, 2007.

[22] J. Beller, M. Heesen, and M. Vollrath, "Improving the driverautomation interaction an approach using automation uncertainty," Human Factors: The Journal of the Human Factors and Ergonomics Society, vol. 55, no. 6, pp. 1130-1141, 2013. 
[23] C. Ahlström and K. Kircher, "Review of real-time visual driver distraction detection algorithms," in Proceedings of the 7th International Conference on Methods and Techniques in Behavioral Research, MB '10, ACM, Eindhoven, The Netherlands, 2010.

[24] B. Donmez, L. N. Boyle, and J. D. Lee, "Safety implications of providing real-time feedback to distracted drivers," Accident Analysis \& Prevention, vol. 39, no. 3, pp. 581-590, 2007.

[25] B. Donmez, L. N. Boyle, and J. D. Lee, "Mitigating driver distraction with retrospective and concurrent feedback," Accident Analysis \& Prevention, vol. 40, no. 2, pp. 776-786, 2008.

[26] H. Zhang, M. Smith, and R. Dufour, "A final report of safety vehicles using adaptive interface technology (phase ii: Task 7c): visual distraction," Tech. Rep., Delphi Electron, Safety, Indiana, Ind, USA, 2008.

[27] T. W. Victor, J. L. Harbluk, and J. A. Engström, "Sensitivity of eye-movement measures to in-vehicle task difficulty," Transportation Research Part F: Traffic Psychology and Behaviour, vol. 8, no. 2, pp. 167-190, 2005.

[28] D. Helbing and B. Tilch, "Generalized force model of traffic dynamics," Physical Review E: Statistical, Nonlinear, and Soft Matter Physics, vol. 58, no. 1, pp. 133-138, 1998.

[29] Y. Li, H. Zhu, M. Cen, Y. Li, R. Li, and D. Sun, "On the stability analysis of microscopic traffic car-following model: A case study," Nonlinear Dynamics, vol. 74, no. 1-2, pp. 335-343, 2013.

[30] K.-T. Chong and D.-H. Roh, "A Lyapunov function approach to longitudinal control of vehicles in a platoon," IEEE Transactions on Vehicular Technology, vol. 50, no. 1, pp. 116-124, 2001.

[31] R. Jiang, Q. Wu, and Z. Zhu, "Full velocity difference model for a car-following theory," Physical Review E: Statistical, Nonlinear, and Soft Matter Physics, vol. 64, no. 1, Article ID 017101, 2001.

[32] H. Liu, D. Sun, and M. Zhao, "Analysis of traffic flow based on car-following theory: a cyber-physical perspective," Nonlinear Dynamics, vol. 84, no. 2, pp. 881-893, 2016. 


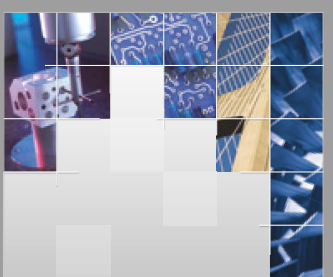

\section{Enfincering}
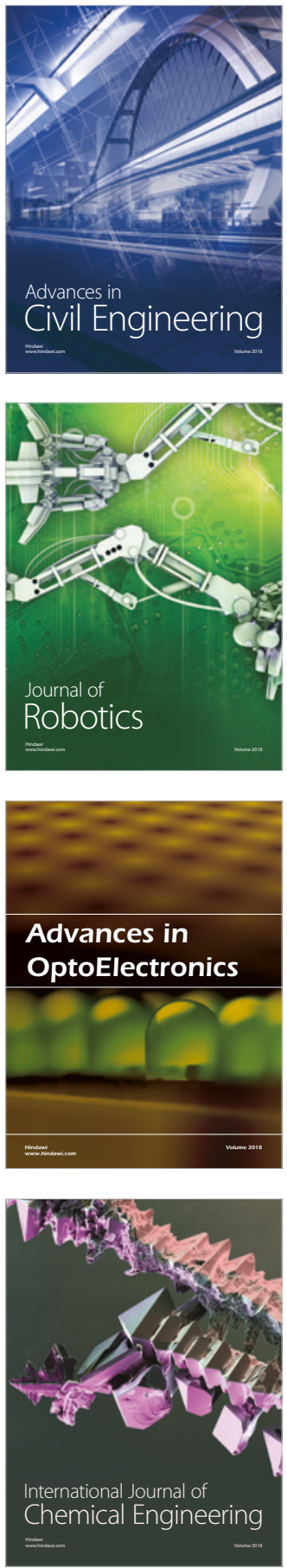

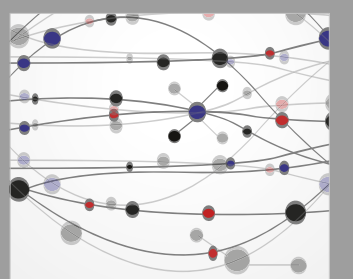

\section{Rotating \\ Machinery}

The Scientific World Journal

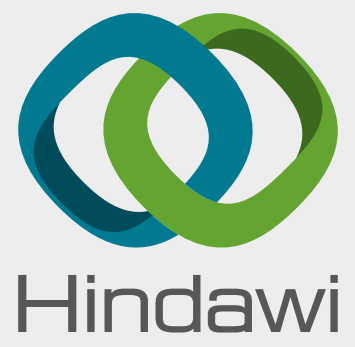

Submit your manuscripts at

www.hindawi.com
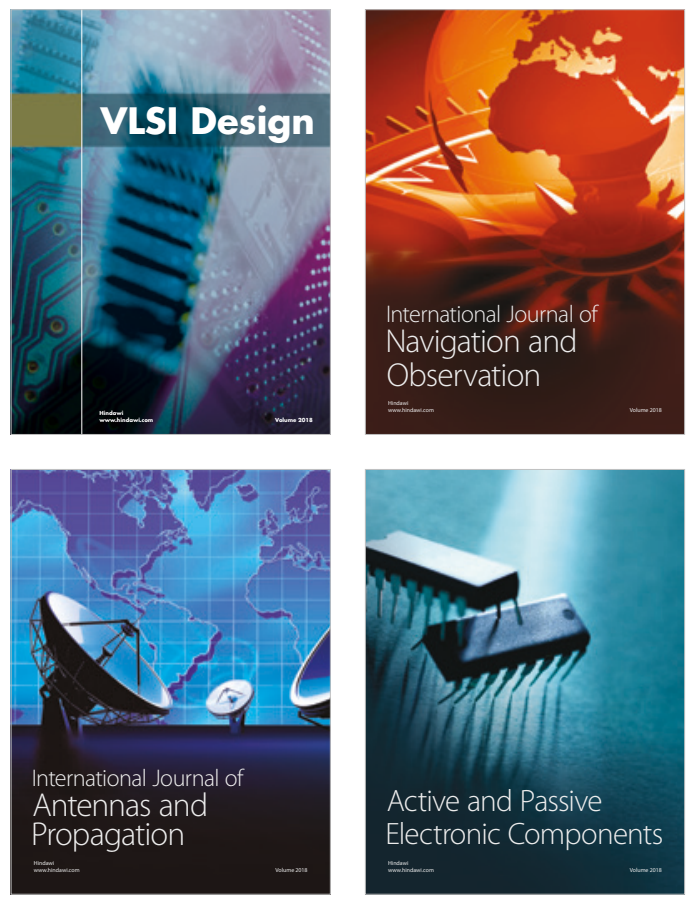
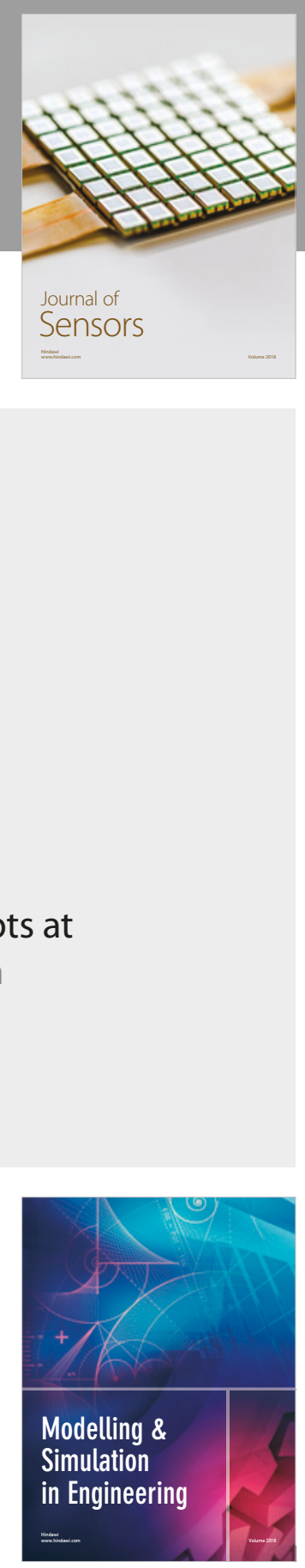

\section{Advances \\ Multimedia}
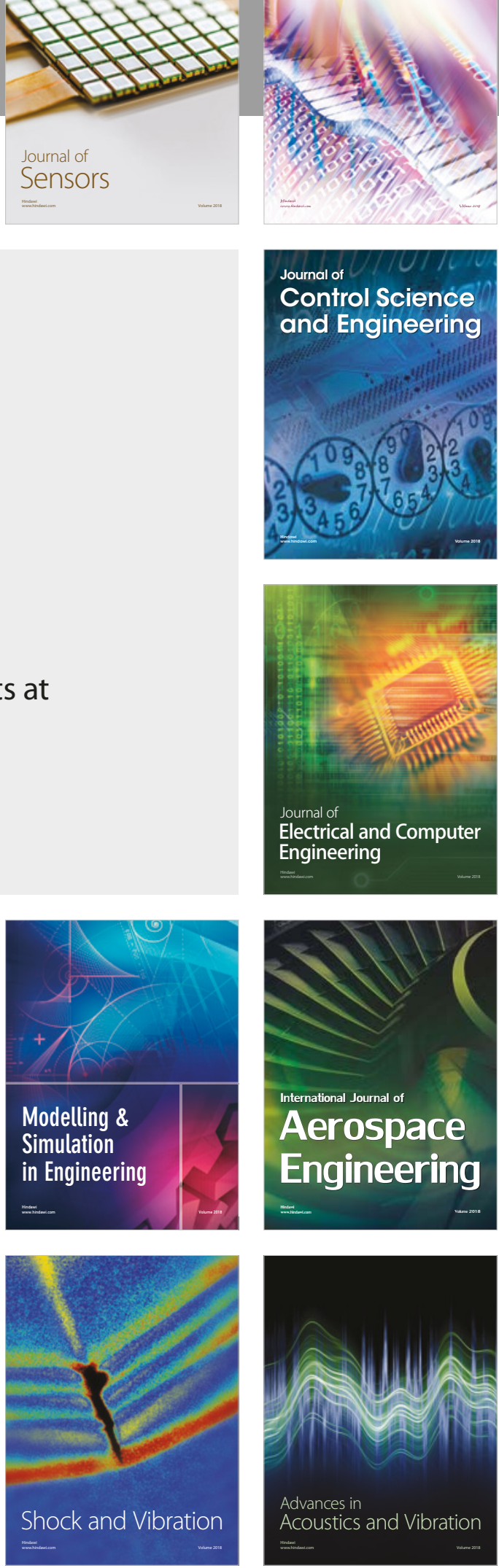